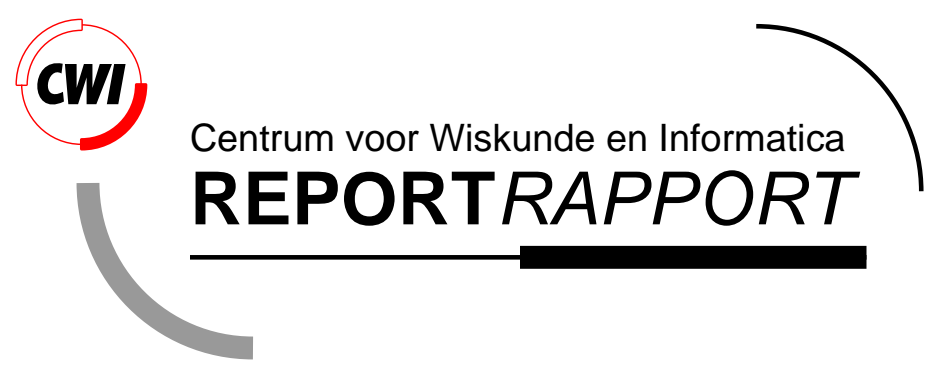

Brine transport in porous media self-similar solutions

C.J. van Duijn, L.A. Peletier, R.J. Schotting

Department of Analysis, Algebra and Geometry

AM-R9616 November 30, 1996 
Report AM-R9616

ISSN 0924-2953

CWI

P.O. Box 94079

1090 GB Amsterdam

The Netherlands

CWI is the National Research Institute for Mathematics and Computer Science. CWI is part of the Stichting Mathematisch Centrum (SMC), the Dutch foundation for promotion of mathematics and computer science and their applications.

SMC is sponsored by the Netherlands Organization for Scientific Research (NWO). CWI is a member of ERCIM, the European Research Consortium for Informatics and Mathematics.

Copyright (C) Stichting Mathematisch Centrum P.O. Box 94079, 1090 GB Amsterdam (NL) Kruislaan 413, 1098 SJ Amsterdam (NL) Telephone +3120 5929333 Telefax +31205924199 


\title{
Brine Transport in Porous Media Self-similar Solutions
}

\author{
C.J. van Duijn \\ CWI
}

P.O. Box 94079, 1090 GB Amsterdam, The Netherlands

email: hansd@cwi.nl

L.A. Peletier

Mathematical Institute, Leiden University

P.O. Box 9512, 2300 RA Leiden, The Netherlands

email: peletier@wi.LeidenUniv.nl

\section{R.J. Schotting ${ }^{1}$ \\ CWI}

P.O. Box 94079, 1090 GB Amsterdam, The Netherlands

email: ruuds@cwi.nl

\begin{abstract}
In this paper we analyze a model for brine transport in porous media, which includes a mass balance for the fluid, a mass balance for salt, Darcy's law and an equation of state, which relates the fluid density to the salt mass fraction. This model incorporates the effect of local volume changes due to variations in the salt concentration. Density variations affect the compressibility of the fluid, which in turn cause additional movement of fluid. Two specific situations are investigated that lead to self similarity. We study the relative importance of the compressibility effect in terms of the relative density difference. Semi-analytical solutions are obtained as well as asymptotic expressions in terms of the relative density difference. It is found that the volume changes have a small but noticeable effect on the mass transport only when the salt concentration gradients are large. Some results on the simultaneous transport of brine and dissolved (radioactive) tracers are presented.
\end{abstract}

AMS Subject Classification (1991): 35K55, 76 S05.

Keywords and Phrases: brine transport, compressibility, similarity transformations, porous media.

\section{Introduction}

Recently Hassanizadeh and Leijnse, [1988] [1990] [1995] revisited the theory of brine transport in porous media, designed numerical codes and did experiments in the laboratory. They raised the question whether (semi-) analytical solutions of the governing equations could be obtained under certain boundary and initial conditions. This question initiated our mathematical study and the results are published in the mathematics literature [Van Duijn et.al.,1992]. Because the subject of brine transport is still of current interest in the hydrological literature and the availability of analytical work in this field is poor, we decided to make the mathematical results more accessible for non-mathematicians and wrote this paper. The material has been extended with new results and the emphasis is now on the construction of semiexplicit self similar solutions.

Brine is water containing a high concentration of salt. In an almost saturated brine the mass fraction $(\omega)$, which is defined as the mass concentration of the salt over the density of the brine, can reach 0.26 . This corresponds to a brine density of approximately $1200 \mathrm{~kg} / \mathrm{m}^{3}$. For sea water $\omega=0.04$ corresponding to a fluid density of $1025 \mathrm{~kg} / \mathrm{m}^{3}$. Mass fraction and density are related by an equation of state, which has been empirically determined. Brines are found in surface waters, such as the Dead Sea, and in groundwater near salt domes [Glasbergen, 1981]. These are geological structures in the subsurface consisting of vertical cylinders of salt, a kilometer or more in diameter, embedded in horizontal or inclined strata. Salt domes are potential places for storage of nuclear waste [Roxburgh, 1987], and it is of practical importance to know the flow of the groundwater around them.

\footnotetext{
${ }^{1}$ Corresponding author
} 
Any model for fluid flow and salt transport in a porous medium must contain the mass balance equations for the fluid and the salt, and Darcy's law. The specific model we propose to study uses the complete fluid balance equation [Hassanizadeh \& Leijnse, 1988]

$$
\phi \frac{\partial \rho}{\partial t}+\operatorname{div}(\rho \mathbf{q})=0,
$$

where $\phi$ denotes the porosity of the medium, $\rho$ the fluid density and $\mathbf{q}$ the specific discharge vector. Introducing the material derivative

$$
\frac{D}{D t}=\frac{\partial}{\partial t}+\frac{\mathbf{q}}{\phi} \cdot \operatorname{grad}
$$

in the balance equation yields

$$
\frac{\phi}{\rho} \frac{D \rho}{D t}+\operatorname{divq}=0 .
$$

This expression shows that density variations may affect the compressibility of the fluid, which in turn can cause an additional movement of the fluid. To make this effect explicit is one of the goals of this study.

In this paper we intend to employ mainly analytical techniques. Therefor we are forced to restrict ourselves in the choice of flow problems. Inspired by earlier work of de Josseling de Jong \& van Duijn [1986] we shall analyze two simplified cases denoted by Problem I and Problem II. Problem I describes the mixing of fresh water and brine, originally separated and parallel flowing, due to transversal dispersion. Problem II relates to the flow of groundwater along the surface of a salt dome. A sketch of the corresponding initial and boundary conditions is given in Figure 1. In Problem I the flow domain is unbounded above

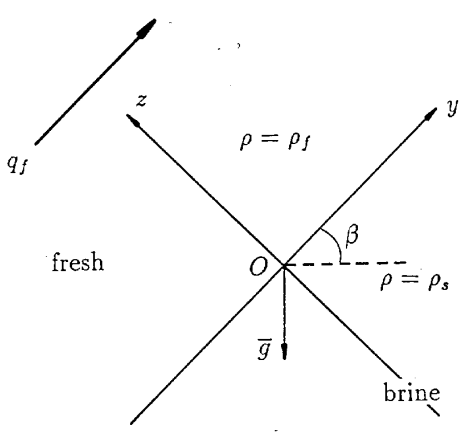

(a) Problem I

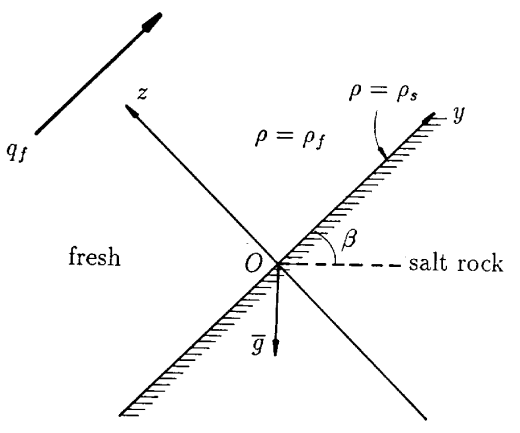

(b) Problem II

Fig 1 . Initial and boundary conditions

and below. Initially, say at $t=0$, the region above the plane $\{z=0\}$ is filled with fresh water and the brine fills the region below it. In this case one has to specify the specific discharge either as $z \rightarrow+\infty$ or as $z \rightarrow-\infty$. Here we shall adopt the former, and fix $\mathbf{q} \rightarrow\left(q_{f}, 0\right)$ as $z \rightarrow+\infty$, where $q_{f}$ is a given constant.

In Problem II the flow domain consists of the upper half space $\{z>0\}$ and is bounded below by an impermeable salt rock. Again at $t=0$, fresh water occupies the region $\{z>0\}$ while the salt from the rock ensures that $\rho=\rho_{s}$ (mass density of saturated brine) along the boundary $\{z=0\}$. Here we can only specify the $y$-component of the specific discharge at $z=+\infty$, because the $z$-component is determined by a second boundary condition, as explained in Section 3, at the surface of the rock. In both problems the $y$-coordinate ranges from $-\infty$ to $+\infty$. 
These problems are chosen because they admit similarity. This means that the underlying partial differential equations can be reduced to ordinary differential equations by introducing an appropriate similarity variable $(z / \sqrt{t}$ in Figure 1). This makes the analysis tractable, yielding semi-explicit results. The mathematical justification of the results is given elsewhere [Van Duijn et.al., 1992]. As a consequence of the analysis we can quantify the effect of the additional brine transport due to the fluid compressibility for Problem I and II, where in particular the latter is relevant to understand the flow near salt domes.

Among recent papers focusing on brine transport we mention [Oldenburg and Pruess,1995; Carey et al.,1995; Herbert et al.,1988; Hassanizadeh and Leijnse, 1995]. Numerical codes [Hassanizadeh and Leijnse, 1990 and 1988] are developed to simulate flow of groundwater containing high salt concentrations. In general it is hard to compare numerical results with experimental data because the availability of brine experiments is poor. However our analytical results can be used to verify the accuracy of numerical codes [HYDROCOIN, 1986, Hassanizadeh, 1990]. Herbert et.al. [1988] stresses the importance of analytical work on this subject.

Hassanizadeh and Leijnse [1995] reported on column tests of brine displacing fresh water in a porous medium. They showed that the dispersivity of brine decreases when the relative density difference between brine and fresh water increases and resolved this problem by introducing a nonlinear form of Fick's law. Carey et al.[1995] suggest a density dependent diffusivity/dispersivity. Although these results are interesting we shall confine ourselves to the classical formulation of Fick's and Darcy's law in this paper.

In Section 2 we formulate the mathematical model in general terms and in Section 3 we define the two specific problems (I and II). Dimensionless variables are introduced in Section 4. In Section 5 we discuss properties and construction of self similar solutions. Numerical procedures and results are given in Section 6 while asymptotic results for small relative density difference, yielding approximately div(q) $=0$, can be found in Section 7. The simultaneous transport of brine and dissolved radionuclides is the subject of Section 8. In Section 9 we discuss the results and Section 10 contains the conclusions.

\section{The mathematical model}

Since this paper focuses on analytical aspects of subsurface brine transport, we shall impose simplifying restrictions on the properties of the porous medium and flow. With respect to the porous medium we assume that it is homogeneous and isotropic, characterized by a constant porosity $\phi$ and intrinsic permeability $\kappa$. With respect to the flow we shall consider the two specific cases which are introduced in Section 1 and about the fluid, with density $\rho$ and salt mass fraction $\omega$, we assume that the dynamic viscosity $\mu$ does not depend on $\omega$ and is constant.

Assuming a Fickian type of dispersion/diffusion term in the salt mass flux and restricting ourselves to the conventional form of the momentum balance equation, we obtain the following equations for transport of brine [Hassanizadeh \& Leijnse, 1986]:

Mass balance of the fluid

$$
\phi \frac{\partial \rho}{\partial t}+\operatorname{div}(\rho \mathbf{q})=0
$$

Mass balance of the salt

$$
\phi \frac{\partial \rho \omega}{\partial t}+\operatorname{div}(\rho \omega \mathbf{q}-D \rho \operatorname{grad} \omega)=0
$$

Darcy's law

$$
\frac{\mu}{\kappa} \mathbf{q}+\operatorname{grad} p-\rho \mathbf{g}=0
$$

Equation of state

$$
\rho=\rho_{f} e^{\gamma \omega} .
$$

Here $\mathbf{q}=\left(q_{y}, q_{z}\right)$ denotes the specific discharge, $p$ the fluid pressure and $\mathbf{g}=\left(g_{y}, g_{z}\right)$ the acceleration of gravity. In the equation of state, where we disregard the effect of pressure variations on the fluid density, $\rho_{f}$ is the density of the fresh water and $\gamma$ is a constant $(\gamma=0.6923 \approx \ln 2)$ obtained by curve fitting 
using a table from Weast [1982]. Following Bear [1972], we use for the hydrodynamic dispersion tensor $D=\left(D_{i j}\right)$ the expression

$$
D_{i j}=\left\{\alpha_{T}|\mathbf{q}|+\phi D_{\text {mol }}\right\} \delta_{i j}+\left(\alpha_{L}-\alpha_{T}\right) q_{i} q_{j} /|\mathbf{q}|,
$$

where $\alpha_{L}$ and $\alpha_{T}$ are positive constants with $\alpha_{L}>\alpha_{T}$. They are called the longitudinal and transversal dispersion length, and describe the spreading of solutes due to mechanical dispersion caused by randomness of the structure of the porous material and heterogeneities. Further $D_{\text {mol }}$ denotes the effective molecular diffusivity, which incorporates the effect of tortuosity. Finally, $\delta_{i j}$ denotes the Kronecker $\delta$ and $|\cdot|$ the Eucidian norm in $\mathbf{R}^{2}$. For mathematical reasons we use in most of this paper (except in Section 9) the approximation

$$
D_{i j}=\phi D \delta_{i j}
$$

where $D$ is a positive constant. If $\alpha_{L}$ and $\alpha_{T}$ are small (fine granular, homogeneous material), then this approximation is justified for $D=D_{m o l}$. However, if the influence of the heterogeneities is significant, then $D$ in (2.6) accounts for dispersion in an averaged sense.

\section{The flow problems}

The object of any study of brine transport is to determine the specific discharge and density (or mass fraction of salt) of the fluid as a function of position and time. We will investigate here two specific problems. In Problem I, see Figure 1(a), we consider an unbounded flow domain above and below the $z=0$ plane. Initially, at time $t=0$ say, the region above this plane is filled with fresh water (density $\rho_{f}$ ) and the region below it with brine (density $\rho_{s}$ ). Since $\rho_{s}>\rho_{f}$, this leads to a stable salt distribution for all $t>0$. As a boundary condition we impose that at large distance above the $z=0$ plane, the flow is known (and given) and points into the $y$-direction:

$$
\mathbf{q} \rightarrow q_{f} \mathbf{e}_{y} \text { as } z \rightarrow+\infty, \text { for all } t \geq 0 .
$$

where $q_{f}$ is a given constant and $\mathbf{e}_{y}$ the unit vector in the positive $y$-direction.

Studies of brine distributions in aquifers have shown that the zone between brine and fresh water is relatively narrow, in particular when the fluids are stagnant. If this situation is perturbed by draining fresh water we end up with a situation that can be represented schematically by Problem I.

In Problem II the flow domain occupies the half space $\{z>0\}$, see Figure 1(b). In formulating this problem we have assumed the initial situation where, due to regional effects, fresh groundwater flows along the top boundary of a salt dome. As a result salt will dissolve from it. The physico-chemical processes that take place at a salt rock boundary are complex and difficult to model. For instance, the dissolution of salt creates a cap (residue) rock layer along the top of the salt dome. Geological studies, e.g. Bornemann et al. [1986], estimate the growth of this layer to be $0.04 \mathrm{~mm} /$ year. Following Hassanizadeh \& Leijnse [1988] we disregard this movement and assume that the mass fraction remains at all times at the maximal salt mass fraction of the saturated brine along the fixed boundary, i.e.

$$
\omega=\omega_{s} \text { at } z=0, \text { for all } t \geq 0 .
$$

Further, the flux of salt entering the flow domain induces a movement of water. This leads to the additional boundary condition, see again Hassanizadeh \& Leijnse [1988],

$$
\left(\mathbf{q}+\frac{D}{\left(1-\omega_{s}\right)} \operatorname{grad} \omega\right) \cdot \mathbf{n}=0 \text { at } z=0, \text { for all } t \geq 0,
$$

where $\mathbf{n}$ denotes the outward normal at the boundary $\{z=0\}$. As initial concentration we have $\rho=\rho_{f}$ or $\omega=0$ everywhere in the flow region. At large distance above the $\{z=0\}$ plane we now impose only the $y$-component of the flow, since the $z$-component will be determined by the problem. Thus we set

$$
q_{y} \rightarrow q_{f} \text { as } z \rightarrow+\infty, \text { for all } t \geq 0 .
$$


In both problems the $y$-coordinate ranges from $-\infty$ to $+\infty$. Therefore we may look for a density and specific discharge depending only on the $z$-coordinate and time, i.e.

$$
\rho=\rho(z, t) \text { and } \mathbf{q}=\mathbf{q}(z, t) .
$$

Following de Josseling de Jong \& van Duijn [1986] we use (3.5) and Darcy's law (2.3) to obtain a linear algebraic relation between the fluid density and the $y$-component of the specific discharge. This relation can be found by first taking the curl of (2.3):

$$
\frac{\partial}{\partial z}\left\{q_{y}-\frac{\kappa}{\mu} \rho g_{y}\right\}-\frac{\partial}{\partial y}\left\{q_{z}-\frac{\kappa}{\mu} \rho g_{z}\right\}=0,
$$

and by substituting (3.5) into this expression. The result is

$$
q_{y}-\frac{\kappa}{\mu} \rho g_{y}=q_{y}+\frac{\kappa}{\mu} \rho g \sin \beta=\text { constant in space, }
$$

where $\beta$ is the inclination of the $z=0$ plane. To determine the constant in (3.7) we use the behavior of $q_{y}$ and $\rho$ at $z=+\infty$ :

$$
q_{y}(\infty, t)=q_{f} \text { and } \rho(\infty, t)=\rho_{f} \text { for all } t>0 .
$$

This yields the relation

$$
q_{y}=q_{f}-\frac{\kappa}{\mu}\left(\rho-\rho_{f}\right) g \sin \beta .
$$

Thus in order to determine the pair $(\rho, \mathbf{q})$ from the differential equations with initial and boundary conditions, there remains, by virtue of (3.9), only to determine $\rho$ and $q_{z}$. Using (3.9), the equations for these quantities reduce to

$$
\phi \frac{\partial \rho}{\partial t}+\frac{\partial}{\partial z}\left(\rho q_{z}\right)=0
$$

and

$$
\phi \frac{\partial \rho \omega}{\partial t}+\frac{\partial}{\partial z}\left(\rho \omega q_{z}-D \rho \frac{\partial \omega}{\partial z}\right)=0 .
$$

Combining these equations yields

$$
\phi \rho \frac{\partial \omega}{\partial t}+\rho q_{z} \frac{\partial \omega}{\partial z}=\frac{\partial}{\partial z}\left(\rho D \frac{\partial \omega}{\partial z}\right)
$$

and with the equation of state (2.4) we obtain

$$
\phi \frac{\partial \rho}{\partial t}+q_{z} \frac{\partial \rho}{\partial z}=D \frac{\partial^{2} \rho}{\partial z^{2}} .
$$

We use the coupled system (3.10) and (3.13) in our analysis. To determine $\rho$ and $q_{z}$ from this system we need to impose the initial and boundary conditions. In Problem I we look for a solution in the domain $\{(z, t):-\infty<z<+\infty, t>0\}$ subject to the initial condition

$$
\rho(z, 0)=\left\{\begin{array}{lll}
\rho_{f} & \text { for } \quad z>0 \\
\rho_{s} & \text { for } \quad z<0
\end{array}\right.
$$

and boundary condition (3.1) for the flow

$$
q_{z}(+\infty, t)=0 \text { for all } t \geq 0
$$

We note that a condition on the flow such as (3.15) is natural for the problem. Combining equations (3.10) and (3.13) gives the ordinary differential equation

$$
\rho \frac{\partial q_{z}}{\partial z}+D \frac{\partial^{2} \rho}{\partial z^{2}}=0
$$


Thus knowing $\rho$, an additional condition on $q_{z}$ is needed to determine the solution. The specific choice is arbitrary. In fact one could construct a solution corresponding to any given $q_{z}(+\infty, t)$. We also observe at this point that equation (3.16), when writing it in the form

$$
\operatorname{divq}=\frac{\partial q_{z}}{\partial z}=-\frac{1}{\rho} \operatorname{div}(D \operatorname{grad} \rho),
$$

clearly demonstrates the coupling between brine transport by diffusion/dispersion, creating a non-zero divergence in the flow field and hence fluid transport by specific discharge. Density gradients imply fluid movement and vice versa.

Problem II is solved in the domain $\{(z, t): z>0, t>0\}$ where we impose initially

$$
\rho(z, 0)=\rho_{f} \text { for } z>0
$$

and along the boundary, see expressions (3.2) and (3.3)

$$
\rho(0, t)=\rho_{s m}:=\rho_{f} e^{\gamma \omega_{s}}
$$

and

$$
q_{z}(0, t)=-\frac{D}{\gamma \rho_{s m}\left(1-\omega_{s}\right)} \frac{\partial \rho}{\partial z}(0, t),
$$

for all $t>0$. Here $\rho_{s m}$ denotes the density of saturated brine. Boundary condition (3.3) relates the specific discharge to the spatial derivative of the fluid density at the salt rock boundary. Although no water is being produced along the salt rock boundary, a non-zero discharge exists along that boundary due to volume effects caused by high salt concentration gradients in the fluid.

\section{Dimensionless variables}

Before discussing equation (3.10) and (3.13) we introduce the dimensionless variables

$$
\rho^{*}=\frac{\rho-\rho_{f}}{\tilde{\rho}_{s}-\rho_{f}}, \quad q_{z}^{*}=\frac{q_{z}}{\frac{\kappa}{\mu} \rho_{f} g}, \quad z^{*}=\frac{\frac{\kappa}{\mu} \rho_{f} g}{D} z, \quad t^{*}=\frac{\left(\frac{\kappa}{\mu} \rho_{f} g\right)^{2}}{\phi D} t
$$

and

$$
\varepsilon=\frac{\tilde{\rho}_{s}-\rho_{f}}{\rho_{f}}
$$

where $\tilde{\rho}_{s}=\rho_{s}$ in Problem I and $\tilde{\rho}_{s}=\rho_{s m}$ in Problem II. In terms of these new variables the equations become (dropping again the asteriks notation)

$$
\frac{\partial \rho}{\partial t}+\frac{\partial}{\partial z}\left(\rho q_{z}\right)+\frac{1}{\varepsilon} \frac{\partial q_{z}}{\partial z}=0
$$

and

$$
\frac{\partial \rho}{\partial t}+q_{z} \frac{\partial \rho}{\partial z}=\frac{\partial^{2} \rho}{\partial z^{2}}
$$

The rescaled initial and boundary conditions in Problem I are

$$
\rho(z, 0)=\left\{\begin{array}{lll}
0 & \text { for } & z>0 \\
1 & \text { for } & z<0
\end{array}\right.
$$

and

$$
q_{z}(+\infty, t)=0 \text { for all } t>0 .
$$

In Problem II the scaling (4.1),(4.2) leads to

$$
\rho(z, 0)=0 \text { for } z>0,
$$




$$
\rho(0, t)=1 \quad \text { for } \quad t>0
$$

and

$$
q_{z}(0, t)=-\varepsilon K(\varepsilon) \frac{\partial \rho}{\partial z}(0, t),
$$

where $K(\varepsilon)$ is given by:

$$
K(\varepsilon)=\frac{\rho_{f}}{\rho_{s m}} \frac{1}{\gamma\left(1-\omega_{s}\right)}=\frac{1}{(1+\varepsilon)(\gamma-\log (1+\varepsilon))} .
$$

Note that the relative density difference lies in the interval

$$
0<\varepsilon<e^{\gamma \omega_{s}}-1 \approx 2^{\omega_{s}}-1 \approx 0.2
$$

for $\omega_{s}=0.26$.

\section{Self-similar solutions}

Due to the nonlinear coupling between equations (4.3) and (4.4) it is not possible to find explicit, closedform solutions of Problems I and II. Nevertheless, their special structure enables us to obtain much information concerning the qualitative behavior of the solutions and to obtain accurate approximations.

The key idea is to look for self-similar solutions, which reduce (4.3),(4.4) to a set of coupled ordinary differential equations with boundary conditions originating from (4.5)-(4.9). The transformed problems were studied in detail by van Duijn et al. [1992]. They considered fundamental questions related to existence and uniqueness of solutions, as well as their qualitative behavior. With respect to the latter, they showed certain monotonicity properties of solutions and their asymptotic behavior for $|z| \rightarrow \infty$ and for $\varepsilon \downarrow 0$. Some of these results will be discussed here and in Section 7 .

The similarity transformations for Problems I and II are given by

$$
\eta=\frac{z}{\sqrt{t}}
$$

and

$$
\rho(z, t)=u(\eta) \text { and } q_{z}(z, t)=\frac{1}{\sqrt{t}} v(\eta)
$$

This results in the set of differential equations

$$
\begin{gathered}
(u v)^{\prime}+\frac{1}{\varepsilon} v^{\prime}-\frac{1}{2} \eta u^{\prime}=0 \\
u^{\prime} v-\frac{1}{2} \eta u^{\prime}=u^{\prime \prime}
\end{gathered}
$$

where the primes denote differentiation with respect to $\eta$. The independent variable $\eta$ ranges from $-\infty$ to $+\infty$ in Problem I and from 0 to $+\infty$ in Problem II.

The following boundary conditions for $u(\eta)$ and $v(\eta)$ in Problem I result from (4.5),(4.6) and are found to be

$$
u(-\infty)=1 \text { and } u(+\infty)=0
$$

and

$$
v(+\infty)=0
$$

For Problem II we find from (4.7)-(4.9)

$$
u(0)=1 \text { and } u(+\infty)=0
$$

and

$$
v(0)=-K(\varepsilon) \varepsilon u^{\prime}(0) .
$$


Equations ( 5.3) and( 5.4) can be combined into:

$$
\begin{gathered}
u^{\prime \prime}+\left(\frac{1}{2} \eta-v\right) u^{\prime}=0, \\
v^{\prime}=-\frac{u^{\prime \prime}}{u+\frac{1}{\varepsilon}} .
\end{gathered}
$$

Let us first discuss Problem I. We start with the important observation that solutions $(u, v)$ of equations (5.9), (5.10) on $\mathbf{R}$ are invariant under linear shifts. By this we mean the following. For any given $a \in \mathbf{R}$, let

$$
\bar{\eta}=\eta-a, \bar{u}(\bar{\eta})=u(\eta) \text { and } \bar{v}(\bar{\eta})=v(\eta)-\frac{1}{2} a .
$$

Then if $(u(\eta), v(\eta))$ solves $(5.9)$ and $(5.10)$ for $-\infty<\eta<+\infty$, than so does $(\bar{u}(\bar{\eta}), \bar{v}(\bar{\eta}))$ if we replace $\eta$ by $\bar{\eta}$ in (5.9). This means that if $(u(\eta), v(\eta))$ is a solution for which $v( \pm \infty)$ exists, then by shifting $\eta$ over a suitable distance $a$, we can reach any limiting value of $v(\eta)$, either at $\eta=-\infty$ or at $\eta=+\infty$. If we choose $a=2 v(+\infty)$ in (5.11) we can ensure that condition (5.6) is satisfied. This invariance property will be used in both the mathematical and numerical procedures for solving Problem I.

Before discussing the construction of solutions, we recall some a priori properties which give an idea about the qualitative behavior of the solution. We proved that

(i) $u^{\prime}(\eta)<0$ for all $-\infty<\eta<+\infty$

(ii) there exists $\eta_{0}>0$ such that $u^{\prime \prime}(\eta)<0$ for $\eta<\eta_{0}$ and $u^{\prime \prime}(\eta)>0$ for $\eta>\eta_{0}$

(iii) $v\left(\eta_{0}\right)=\frac{1}{2} \eta_{0}$ and $v^{\prime}(\eta)>0$ for $\eta<\eta_{0}$ and $v^{\prime}(\eta)<0$ for $\eta>\eta_{0}$

(iv) $u^{\prime}(\eta) \rightarrow 0$ if $|\eta| \rightarrow \infty$

The first assertion implies that the brine concentration increases strictly with depth and is concave below the plane $z=\eta_{0} \sqrt{t}$ and convex above it. Also the $z$-component of the specific discharge has a maximum at $z=\eta_{0} \sqrt{t}$ of magnitude $q_{z}\left(\eta_{0} \sqrt{t}, t\right)=\frac{1}{2} \eta_{\mathrm{o}} / \sqrt{t}$. The number $\eta_{\mathrm{o}}$ plays a prominent role in simultaneous transport of brine and radionuclides, which will be explained in Section 8 .

Next we turn to the solution procedure. As a first observation we note that equations (5.9)-(5.11) can be combined into a single equation with $\eta$ missing. This equation, having the form

$$
\frac{1}{2}+\left(\frac{u^{\prime \prime}}{u^{\prime}}\right)^{\prime}=-\frac{u^{\prime \prime}}{u+\frac{1}{\varepsilon}} \text { with }-\infty<\eta<+\infty,
$$

needs three conditions to be solved uniquely. Two conditions are given by (5.5). As a third condition we take, for instance,

$$
u(0)=\frac{1}{2} .
$$

We outline below how to obtain a unique solution satisfying (5.12), (5.5) and (5.13) and how to obtain from that solution a corresponding $v=v(\eta)$ so that the pair $(u, v)$ satisfies equations (5.3) and (5.4). This function $v$ will not satisfy condition (5.6). To achieve that condition one applies the linear shift (5.11) with $a=2 v(\infty)$.

Equation (5.12) is of third order, nonlinear and defined on an unbounded domain. To tackle it directly is therefor not straightforward. As so often in mathematics, we solve problems by combining and applying what we already know. Also in this case. We will transform equation (5.12) into a second order equation on a finite domain, yielding a boundary value problem which is well-known in the mathematical literature. This transformation is achieved by taking $u$ as the independent variable, which is allowed by the monotonicity of $u$, and by taking $u^{\prime}$ as the new independent variable. Thus setting

$$
\eta=\eta(u) \text { and } y(u)=-u^{\prime}(\eta(u)) .
$$

we obtain

$$
y\left\{(1+\varepsilon u) y^{\prime}\right\}^{\prime}=-\frac{1}{2}(1+\varepsilon u), \quad 0<u<1
$$


In view of property (iv) we obtain for $y$ the boundary conditions

$$
y(0)=0 \quad \text { and } \quad y(1)=0 .
$$

By setting further

$$
s=\frac{\log (1+\varepsilon u)}{\log (1+\varepsilon)} \quad \text { and } \quad z(s)=\frac{\varepsilon}{\log (1+\varepsilon)} y(u)
$$

we obtain the problem

$$
\left(I^{\prime}\right)\left\{\begin{array}{l}
-z z^{\prime \prime}=\frac{1}{2} e^{2 s \log (1+\varepsilon)}, \quad z>0 \text { for } 0<s<1 \\
z(0)=z(1)=0 .
\end{array}\right.
$$

which is well-known and arises in the study of self-similar solutions of nonlinear diffusion equations, see for instance Esteban et.al. [1988], van Duijn \& Peletier [1977] and van Duijn \& Floris [1991]. In the context of nonlinear diffusion one calls equation (5.18) the flux equation, because it specifies the flux in terms of the concentration. One sees immediately (since $z>0$ ) that

$$
z^{\prime \prime}(s)<0 \text { for } 0<s<1 .
$$

Less straightforward are the proofs of the properties

$$
\lim _{s \downarrow 0} z^{\prime}(s)=+\infty \text { and } \lim _{s \uparrow 1} z^{\prime}(s)=-\infty,
$$

which can be found in van Duijn \& Floris [1991]. Knowing that a solution $z=z(s)$ of Problem $I^{\prime}$ exists and is unique, and knowing much about its qualitative behavior, one finds $y=y(u)$ from (5.17). Finally, the solution $u=u(\eta)$ satisfying (5.12),(5.5) and (5.13) is implicitly defined by

$$
\eta(u)=\int_{u}^{\frac{1}{2}} \frac{1}{y(s)} d s
$$

which results from integrating ( 5.14). The corresponding $v=v(\eta)$ is given by

$$
v(\eta)=\frac{1}{2} \eta-y^{\prime}(u(\eta)) \text { for }-\infty<\eta<+\infty
$$

which follows from ( 5.9) and (5.14).

Since Problem II is defined on the semi-infinite interval $0<\eta<+\infty$, we loose invariance property (5.11). But the other properties (i)..(iv) remain the same, when taking $0<\eta<+\infty$, yielding a similar qualitative picture of the solution. The solution procedure proceeds along the same lines. It leads to the transformed problem

$$
\left(I I^{\prime}\right)\left\{\begin{array}{l}
-z z^{\prime \prime}=\frac{1}{2} e^{2 s \log (1+\varepsilon)}, \quad z>0 \text { for } 0<s<1 \\
z(0)=0, \quad z^{\prime}(1)=-L z(1),
\end{array}\right.
$$

where $L$ is a constant given by

$$
L=K(\varepsilon)(1+\varepsilon) \log (1+\varepsilon)=\frac{\log (1+\varepsilon)}{\gamma-\log (1+\varepsilon)} .
$$

The boundary condition at $s=1$ is a direct consequence of condition (5.8). To return to $u=u(\eta)$, we first use again (5.17) to obtain $y=y(u)$ and then

$$
\eta=\int_{u(\eta)}^{1} \frac{1}{y(s)} d s \text { for } 0<\eta<+\infty
$$

The corresponding $v=v(\eta)$ is obtained from (5.22). 


\section{Numerical procedures and results}

The mathematical analysis has provided us with qualitative information about the structure of the solutions of Problem I and II. This information can be used to develop procedures to obtain accurate numerical solutions.

Starting point for both problems is the third order equation (5.12). We write this equation as a system of first order equations by setting

$$
p=u, q=u^{\prime} \text { and } r=v .
$$

Substitution in (5.12) yields

$$
(S)\left\{\begin{aligned}
p^{\prime} & =q \\
q^{\prime} & =q\left(r-\frac{1}{2} x\right) \\
r^{\prime} & =-\frac{q\left(r-\frac{1}{2} x\right)}{p+\frac{1}{\varepsilon}} .
\end{aligned}\right.
$$

To solve this system we impose three conditions at $\eta=0$ :

$$
p(0)=u(0)=p_{0}, \quad q(0)=u^{\prime}(0)=q_{0}, \quad r(0)=v(0)=r_{0},
$$

where $p_{0}=1 / 2$ (Problem I) or $p_{0}=1$ (Problem II), and where $q_{0}$ and $r_{0}$ are a priori unknown. They have to be determined from the boundary conditions (5.5) (Problem I) or from (5.7), (5.8) (Problem II). Concerning Problem I, one approach is to apply a shooting procedure in the regions $\{\eta<0\}$ and $\{\eta>0\}$. Taking for $(S)$ the initial values $p_{0}=1 / 2$ and $q_{0}, r_{0}$ arbitrary, one solves the equations for $\{\eta<0\}$ and $\{\eta>0\}$. The idea is to choose $p_{0}$ and $q_{0}$ such that

$$
p(-\infty)=u(-\infty)=1 \text { and } p(+\infty)=u(+\infty)=0 .
$$

However, this procedure is not at all trivial because there are two degrees of freedom in the problem. Moreover it would be time consuming to compute accurate values for $q_{0}$ and $r_{0}$ by trail and error such that the correct limiting behavior for $u$ at $\eta=+/-\infty$ is achieved.

An alternative approach is to determine $q_{0}$ and $r_{0}$ directly from Problem $\left(I^{\prime}\right)$ by noting that

$$
p_{0}=-y\left(\frac{1}{2}\right)=\frac{\log (1+\varepsilon)}{\varepsilon} z\left(\frac{\log \left(1+\frac{\varepsilon}{2}\right)}{\log (1+\varepsilon)}\right)
$$

and, using (5.8),

$$
r_{0}=-y^{\prime}\left(\frac{1}{2}\right)=\frac{1}{1+\frac{\varepsilon}{2}} z^{\prime}\left(\frac{\log \left(1+\frac{\varepsilon}{2}\right)}{\log (1+\varepsilon)}\right) .
$$

We find approximate values of the shooting parameters $q_{0}$ and $r_{0}$ by solving $\left(I^{\prime}\right)$ numerically. We omit the details of the computations. Next equations (S) are solved using a fourth order Runge-Kutta method in the regions $\{\eta<0\}$ and $\{\eta>0\}$, subject to $p_{0}, q_{0}$ and $r_{0}$. This gives the solution $(u(\eta), v(\eta))$ of $(5.9)$ and (5.10) which satisfies $u(0)=\frac{1}{2}$. It also provides us with the value of $v(+\infty)=r(+\infty)$ which we need to obtain the correct shift $a=2 v(+\infty)$ to satisfy boundary condition (5.6).

In all cases considered, i.e. for all relevant values of $\varepsilon$, we verified the boundary conditions $u(+\infty)=0$ and $u(-\infty)=1$. Up to a small error term these values are taken on by the numerical approximations. This serves as an independent check for the accuracy of the numerical procedure for Problem (I').

Figures 2 and 3 give the results of the numerical approximations of the similarity solutions $u(\eta)$ and $v(\eta)$ for different values of the relative density difference $\varepsilon$. 


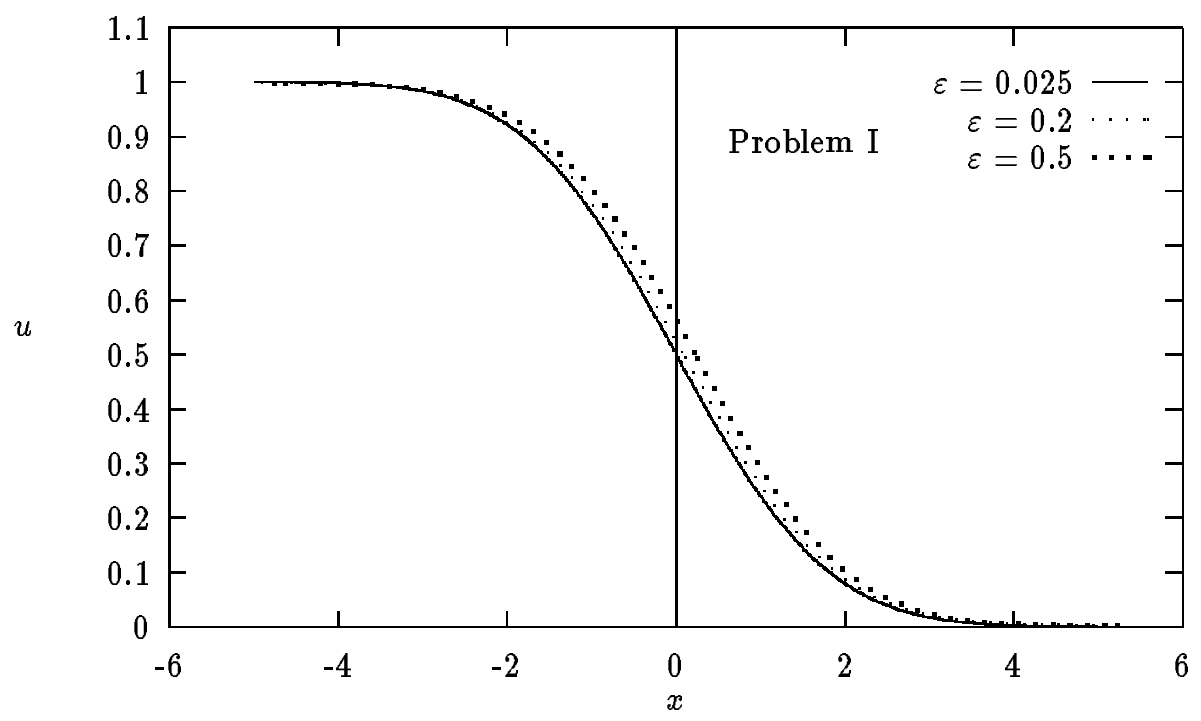

Figure 2. Numerical approximations of $u(\eta)$ for Problem I.

The value $\varepsilon=0.025$ corresponds to sea water. This curve is hardly distinguishable from the curve for $\varepsilon=0$ on the scale of Figure 2. The limit $\varepsilon \rightarrow 0$ is the so-called Boussinesq approximation or Boussinesq limit, see Section 7. The value $\varepsilon=0.2$ corresponds with an almost saturated brine, while $\varepsilon=0.5$ is chosen here to emphasize the effect. Observe that the numerical solutions satisfy the qualitative behavior discussed in Section 5.

Concerning Problem II we solve equations (S) for $\eta>0$ subject to the initial conditions

$$
\begin{aligned}
& p(0)=u(0)=1, \\
& q(0)=u^{\prime}(0)=q_{0}<0, \\
& r(0)=v(0)=-\varepsilon K(\varepsilon) q_{0},
\end{aligned}
$$

where the last condition is a consequence of (5.8). This is a one-parameter shooting problem which is more straightforward and is solved without using Problem (II'). The object is to find a approximate value of $q_{0}$ such that the boundary condition $u(+\infty)$ is satisfied. This can be achieved by starting the shooting procedure with an initial estimate for $q_{0}$ and check if the corresponding limiting behavior is satisfied at sufficiently large distance $L$ from the origin.

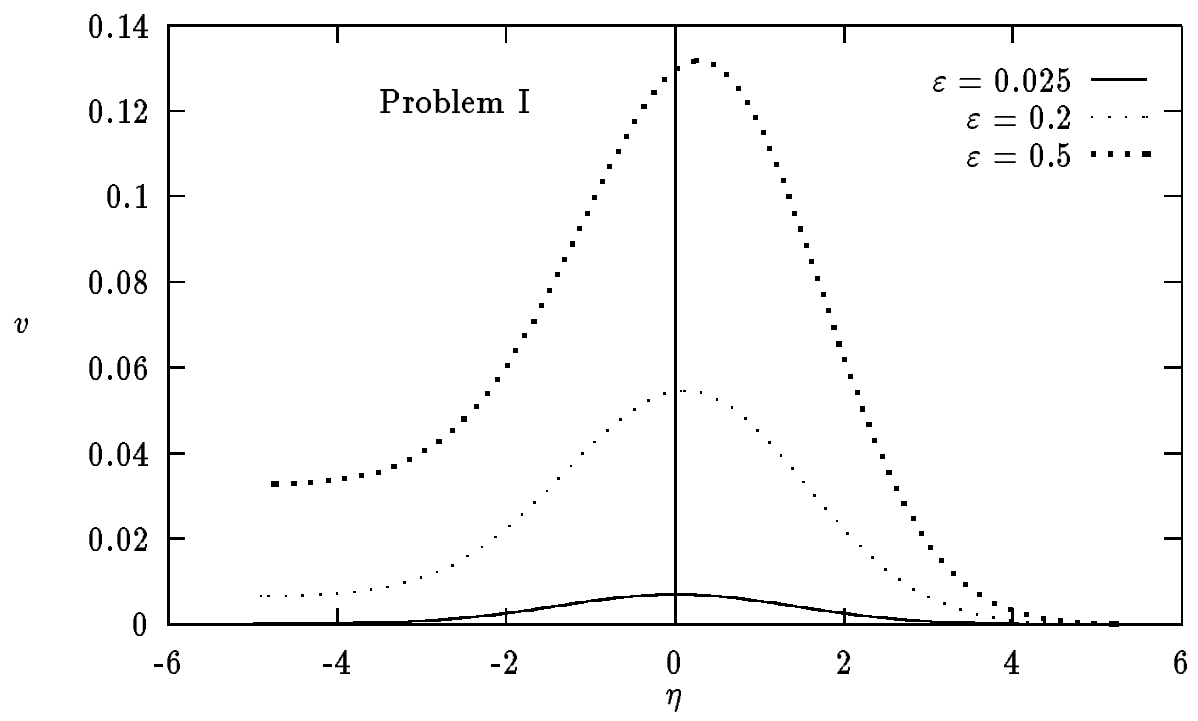

Figure 3. Numerical approximations of $v(\eta)$ for Problem I. 
The problem is to determine an approximate value of $q_{0}$ such that the boundary condition $u(+\infty)=0$ is satisfied. This can be achieved by starting the shooting procedure with an estimated value of $q_{0}$ and check if the corresponding limiting behavior is satisfied at a sufficiently large distance $L$ from the origin. If $u(L)>\delta>0$, where $\delta$ an a priori specified small error term, the estimated value of $q_{0}$ is decreased by a fixed amount $\Delta q_{0}$ and the shooting procedure is repeated. The step size $\Delta q_{0}$ remains fixed until, say after the $n$-the step, $u^{n}(L)<0$. Now $q_{0}^{n}$ is increased by the bisection of $\Delta q_{0}$, hence $q_{0}^{n+1}=q_{0}^{0}-\Delta q_{0}\left(n-\frac{1}{2}\right)$. If $u^{n+1}(L)>\delta$ or if $u^{n+1}(L)<0$ we bistect the last alteration of $q_{0}$ again and obtain respectively : $q_{0}^{n+2}=q_{0}^{0}-\Delta q_{0}\left(n-\frac{1}{4}\right)$ or $q_{0}^{n+2}=q_{0}^{0}-\Delta q_{0}\left(n-\frac{3}{4}\right)$. This procedure is repeated until $0 \leq u(L) \leq \delta$. The number of steps depends on the quality of the initial guess of $q_{0}$ and the initial step size $\Delta q_{0}$.

Figure 4 shows the results of the iterative shooting procedure for the scaled density $u(\eta)$ for different $\varepsilon$-values. The corresponding scaled specific discharge distributions $v(\eta)$ are given in Figure 5. Notice that $v(0) \neq 0$. This is a consequence of boundary condition (5.8) at the salt rock/brine interface. Because $u$ is a decreasing function we have $u^{\prime}(0)<0$, hence $v(0)>0$, for all $\varepsilon>0$.

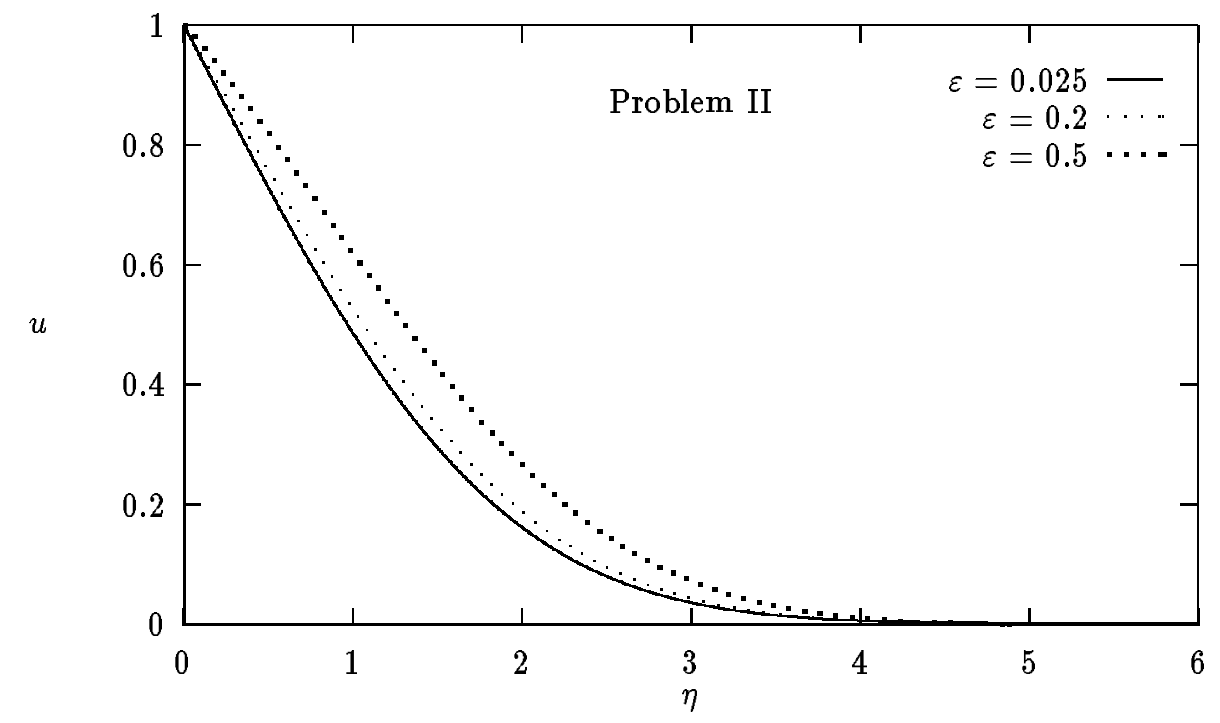

Figure 4. Numerical approximations of $u(\eta)$ for Problem II.

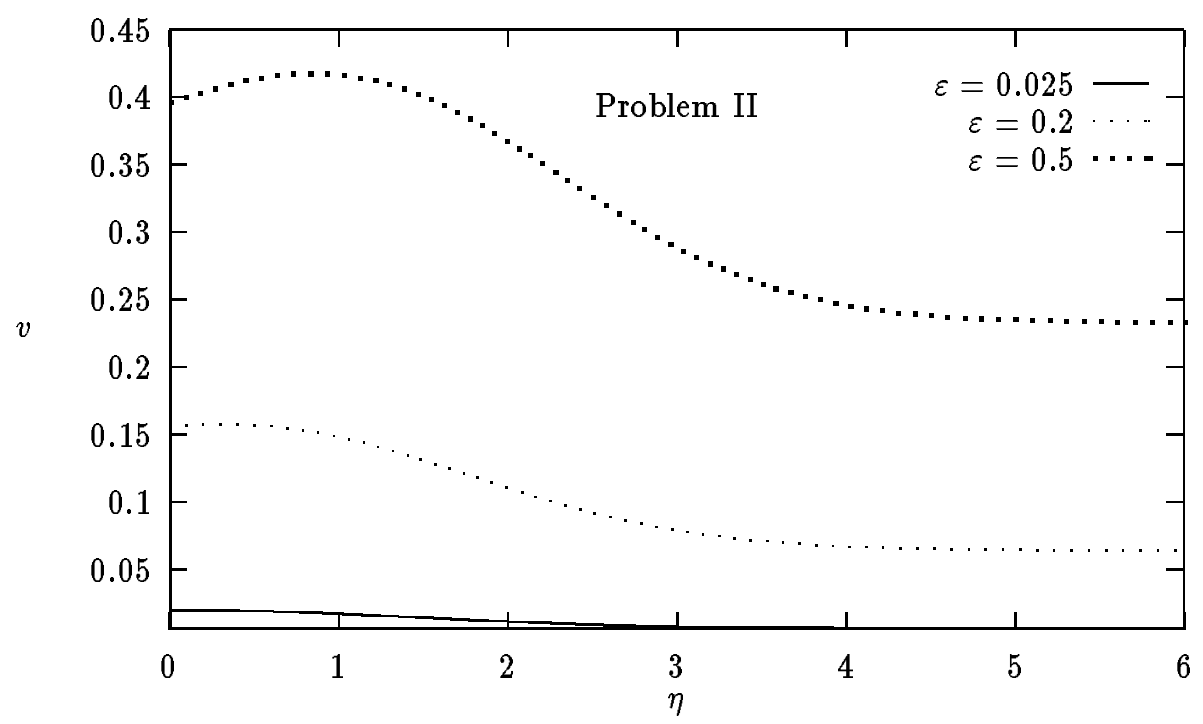

Figure 5. Numerical approximations of $v(\eta)$ for Problem II.

Figures 6 and 7 give the relative density $\rho(z, t)=u(z / \sqrt{t})$ and the unscaled specific discharge $q_{z}(z, t)=$ 
$v(z / \sqrt{t}) / \sqrt{t}$ at different time levels in the original variables. The other parameter values used in these examples are adopted from Herbert et al. [1988] and listed in Table 1.

\begin{tabular}{|l|l|l|}
\hline Property & Value & Dimension \\
\hline$\kappa$ & $10^{-12}$ & $\mathrm{~m}^{2}$ \\
$\mu$ & $10^{-3}$ & Pas \\
$g$ & 9.81 & $\mathrm{~m} / \mathrm{s}^{2}$ \\
$\phi$ & 0.3 & - \\
$\rho_{f}$ & 1000 & $\mathrm{~kg} / \mathrm{m}^{3}$ \\
$D$ & $10^{-9}$ & $\mathrm{~m}^{2} / \mathrm{s}$ \\
\hline
\end{tabular}

Table 1.

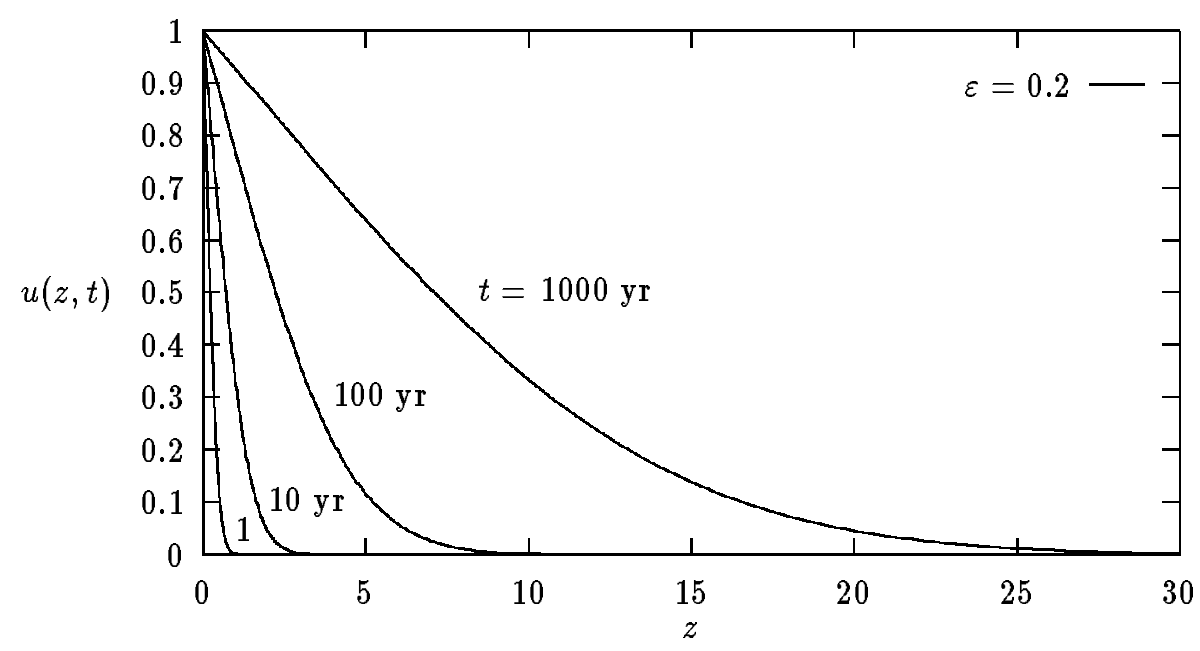

Figure 6. Brine density profiles at different times for $\varepsilon=0.2$ in Problem II

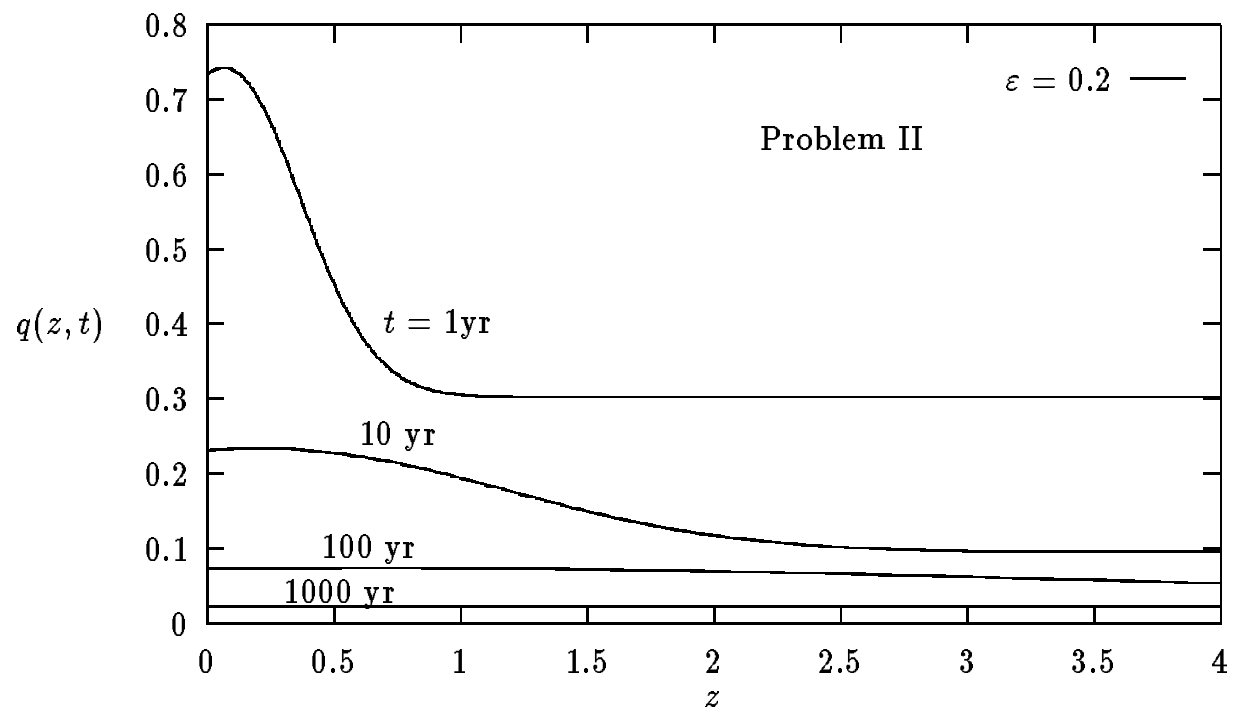

Figure 7. Specific discharge at different times for $\varepsilon=0.2$ in Problem II.

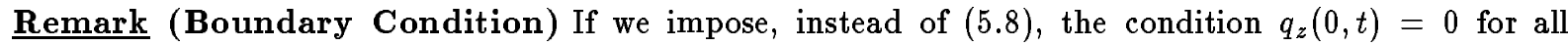
$t>0$ to express the assumption that the salt rock boundary is impervious, then the analysis yields 
$v(\eta)<v(0)=0$ for all $\eta>0$. An explanation for this behavior is that $q_{z}=0$ at the boundary can only be maintained by a back flow coming from $+\infty$. We conclude that this is a physically unrealistic situation and that the no flow boundary is incompatible with the model discussed in this paper.

\section{Approximate self similar solutions}

In this section we give some results of a formal asymptotic analysis which can be found in detail in van Duijn et al. [1992]. The asymptotic analysis yields series expansions in terms of the relative density difference $\varepsilon$ for the similarity solutions $(u(\eta), v(\eta))$ in both problems. When these expansions are truncated we obtain approximation formulas which can be of practical use to approximate $(u(\eta), v(\eta))$ up to a certain, known accuracy. To emphasize the dependence on $\varepsilon$, we denote the solutions by $\left(u_{\varepsilon}(\eta), v_{\varepsilon}(\eta)\right)$. In the limit $\varepsilon \rightarrow 0$ the solutions $\left(u_{\varepsilon}, v_{\varepsilon}\right)$ converge to the corresponding Boussinesq limit $\left(u_{0}, v_{0}\right)$ for both problems. For Problem I $v_{0}=0$, while the limit for $u_{0}$ is the solution of

$$
B_{I}\left\{\begin{array}{l}
u_{0}^{\prime \prime}+\frac{1}{2} \eta u_{0}^{\prime}=0 \text { for }-\infty<\eta<+\infty \\
u_{0}(-\infty)=1 \text { and } u_{0}(+\infty)=0
\end{array}\right.
$$

and is given by

$$
u_{0}(\eta)=\frac{1}{2} \operatorname{erfc}\left(\frac{\eta}{2}\right)
$$

If we integrate $(5.10)$ over $(\eta,+\infty)$ and apply the boundary condition $v(+\infty)=0$ we obtain

$$
v(\eta)=-\varepsilon \frac{u^{\prime}(\eta)}{(1+\varepsilon u(\eta))}+\varepsilon^{2} \int_{\eta}^{+\infty} \frac{\left(u^{\prime}(s)\right)^{2}}{(1+\varepsilon u(s))^{2}} d s .
$$

By (7.3) we have

$$
\lim _{\varepsilon \rightarrow 0} \frac{v(\eta)}{\varepsilon}=-u_{0}^{\prime}(\eta)
$$

This expression can be used as a first order approximation of $v_{\varepsilon}(\eta)$. We can improve the quality of the approximation by adding more terms in the expansion

$$
v_{\varepsilon}(\eta)=-u_{0}(\eta) \varepsilon+\left\{E_{1}(\eta)+o(1)\right\} \varepsilon^{2}
$$

where

$$
E_{1}(\eta)=2 u_{0}(\eta) u_{0}^{\prime}(\eta)-\frac{1}{2} u_{0}^{\prime}(\eta)+\int_{\eta}^{\infty}\left\{u_{0}^{\prime}(s)\right\}^{2} d s
$$

The symbol $o(1)^{2}$ denotes the order symbol. A similar series expansion for $u_{\varepsilon}(\eta)$ can be derived

$$
u_{\varepsilon}(\eta)=u_{0}(\eta)+\frac{1}{2} u_{0}(\eta)\left\{1-u_{0}(\eta)\right\} \varepsilon+\left\{E_{2}(\eta)+o(1)\right\} \varepsilon^{2},
$$

where $E_{2}(\eta)$ is given by

$$
E_{2}(\eta)=\int_{\eta}^{\infty} e^{-t^{2} / 4} \int_{0}^{t} e^{s^{2} / 4} f_{\varepsilon}(s) d s d t-A_{\varepsilon} \int_{\eta}^{\infty} e^{-t^{2} / 4} d t
$$

In the latter expression $f_{\varepsilon}(\eta)$ and $A_{\varepsilon}$ are

$$
f_{\varepsilon}(\eta)=\left(1-3 u_{0}(\eta)\right)\left(u_{0}^{\prime}(\eta)\right)^{2}-u_{0}^{\prime}(\eta) \int_{\eta}^{\infty}\left\{u_{0}^{\prime}(s)\right\}^{2} d s
$$

\footnotetext{
${ }^{2}$ The expression $f(x)=o(\psi(x))$ for $x \rightarrow+\infty$ means that $f(x) / \psi(x)$ tends to zero when $x \rightarrow+\infty$. It can be read as: 'Something that tends to zero, multiplied by'.
} 


$$
A_{\varepsilon}=\frac{1}{2 \sqrt{\pi}} \int_{-\infty}^{\infty} e^{-t^{2} / 4} \int_{0}^{t} e^{s^{2} / 4} f_{\varepsilon}(s) d s d t
$$

Both series expansions are truncated after the $\varepsilon^{2}$ terms. A nice property of the approximate solutions $u_{\varepsilon}$ and $v_{\varepsilon}$ is that they are expressed in terms of the solution of the Boussinesq problem $B_{I}$ and the small parameter $\varepsilon$ only. Because $u_{0}(\eta)$ is known explicitly, $u_{\varepsilon}$ and $v_{\varepsilon}$ can be evaluated without great difficulty, for instance with the computer algebra package Maple [1994].

In the Boussinesq limit for Problem II we have again $v_{0}=0$ while now $u_{0}$ is the solution of

$$
B_{I I}\left\{\begin{array}{l}
u_{0}^{\prime \prime}+\frac{1}{2} \eta u_{0}^{\prime}=0 \text { for } \eta>0 \\
u(0)=1 \text { and } u(+\infty)=0
\end{array}\right.
$$

hence

$$
u_{0}(\eta)=\operatorname{erfc}\left(\frac{\eta}{2}\right)
$$

The asymptotic expressions for Problem II are

$$
v_{\varepsilon}(\eta)=\left\{\frac{(1-\gamma)}{\sqrt{\pi} \gamma}-u_{0}^{\prime}(\eta)\right\} \varepsilon+\left\{E_{3}(\eta)+o(1)\right\} \varepsilon^{2}
$$

and

$$
u_{\varepsilon}(\eta)=u_{0}(\eta)-\left\{\frac{2(1-\gamma)}{\pi \gamma}\left\{u_{0}(\eta)+\sqrt{\pi} u_{0}^{\prime}(\eta)\right\}+\frac{1}{2} u_{0}(\eta)\left\{1-u_{0}(\eta)\right\}\right\} \varepsilon+\left\{E_{4}(\eta)+o(1)\right\} \varepsilon^{2}
$$

with

$$
E_{3}(\eta)=\frac{\gamma^{2}-\gamma+1}{\sqrt{\pi} \gamma}+u_{0}^{\prime}(\eta)\left(2 u_{0}(\eta)-\frac{\gamma(\pi+4)-4}{2 \gamma \pi}\right)-\frac{2(\gamma-1)}{\gamma \sqrt{\pi}} u_{0}^{\prime \prime}(\eta)+\int_{\eta}^{+\infty}\left\{u_{0}^{\prime}(s)\right\}^{2} d s
$$

and

$$
E_{4}(\eta)=A_{\varepsilon} \int_{0}^{\eta} e^{-t^{2} / 4} d t-\int_{0}^{\eta} e^{-t^{2} / 4} \int_{0}^{t} e^{s^{2} / 4} f_{\varepsilon}(s) d s d t
$$

The function $f_{\varepsilon}(\eta)$ and the constant $A_{\varepsilon}$ are defined as

$$
f_{\varepsilon}(\eta)=\left(\frac{1}{2} u_{0}(\eta)\left(1-u_{0}(\eta)\right)+\frac{2(\gamma-1)}{\gamma \pi}\left(u_{0}(\eta)+\sqrt{\pi} u_{0}^{\prime}(\eta)\right)\right)\left(u_{0}^{\prime}(\eta)-\frac{1-\gamma}{\gamma \sqrt{\pi}}\right)-u_{0}^{\prime}(\eta) E_{3}(\eta)
$$

and

$$
A_{\varepsilon}=\frac{1}{\sqrt{\pi}} \int_{0}^{\infty} e^{-t^{2} / 4} \int_{0}^{t} e^{s^{2} / 4} f_{\varepsilon}(s) d s d t
$$

\section{Transport of radionuclides}

In this section we consider the simultaneous transport of radionuclides, occurring in tracer concentrations, in the vicinity of a salt dome. We assume that the flow geometry is given by Problem II, resulting in one-dimensional ( $z$-direction) transport. The equation to be solved is

$$
\phi \frac{\partial \rho \omega_{c}}{\partial t}+\frac{\partial}{\partial z}\left(\rho \omega_{c} q_{z}-D_{c} \rho \frac{\partial \omega_{c}}{\partial z}\right)-\phi \lambda \rho \omega_{c}=0
$$

where $\omega_{c}$ denotes the mass fraction of a radionuclide, $\lambda$ the decay constant and $D_{c}$ its effective diffusivity/dispersivity. Note that $q_{z}=q_{z}(z, t)$ and $\rho=\rho(z, t)$ are solutions of Problem II. Further note that in general $D_{c} \neq D$, the diffusivity of the salt. We introduce the parameter $\theta$, defined as

$$
\theta=D_{c} / D
$$


and apply the scaling rules (4.1) in (8.1). This ensures identical time scales for brine and radionuclide transport. The radionuclide mass fraction is scaled according to

$$
\omega_{c}^{*}=\frac{\omega_{c}}{\omega_{0}}
$$

where $\omega_{0}$ denotes a reference mass fraction, e.g. the (radionuclide) mass fraction at the salt rock boundary. As a consequence of (4.1) we find

$$
\lambda^{*}=\frac{\phi D}{\left(\frac{\kappa}{\mu} \rho_{f} g\right)^{2}} \lambda
$$

as the dimensionless decay constant. Combining (8.1) and (3.10), and applying (4.1), (8.2), (8.3) and (8.4), we obtain (omitting the asteriks again)

$$
\frac{\partial \bar{\omega}_{c}}{\partial t}+q_{z} \frac{\partial \bar{\omega}_{c}}{\partial z}-\frac{\theta}{(\varepsilon \rho+1)} \frac{\partial}{\partial z}\left((\varepsilon \rho+1) \frac{\partial \bar{\omega}_{c}}{\partial z}\right)=0 .
$$

in which we eliminated the decay term in the usual way, i.e. by setting $\omega_{c}(z, t)=\bar{\omega}_{c}(z, t) e^{-\lambda t}$. The boundary and initial conditions are

$$
\begin{array}{lll}
\bar{\omega}_{c}(0, t)=1 & \text { for all } & t>0 \\
\bar{\omega}_{c}(z, 0)=0 & \text { for all } & z>0
\end{array}
$$

Since $q_{z}(z, t)=v(\eta) / \sqrt{t}$ and $\rho(z, t)=u(\eta)$, we note that (8.5)-(8.6) has a self similar solution $\bar{\omega}_{c}(z, t)=$ $f(\eta)$, where $f$ is a solution of the linear boundary value problem

$$
(B V P)\left\{\begin{array}{l}
f^{\prime}\left(v-\frac{\eta}{2}\right)-\frac{\theta}{(\varepsilon u+1)}\left((\varepsilon u+1) f^{\prime}\right)^{\prime}=0 \\
f(0)=1 \\
f(\infty)=0
\end{array}\right.
$$

To solve this problem we first eliminate $v$ using (5.9) and next integrate the resulting equation. This leads to

$$
f(\eta)=1-\frac{\int_{0}^{\eta}\left(\frac{u^{\prime}(\xi)}{u^{\prime}(0)}\right)^{\frac{1}{\theta}} \frac{(1+\varepsilon)}{\varepsilon u(\xi)+1)} d \xi}{\int_{0}^{\infty}\left(\frac{u^{\prime}(\xi)}{u^{\prime}(0)}\right)^{\frac{1}{\theta}} \frac{(1+\varepsilon)}{\varepsilon u(\xi)+1)} d \xi}=1-\frac{\int_{0}^{\eta} \frac{\left|u^{\prime}(\xi)\right|^{\frac{1}{\theta}}}{\varepsilon u(\xi)+1} d \xi}{\int_{0}^{\infty} \frac{\left|u^{\prime}(\xi)\right|^{\frac{1}{\theta}}}{\varepsilon u(\xi)+1} d \xi}
$$

where $u$ is the solution of Problem II, for a given value of $\varepsilon$. The corresponding, scaled radionuclide concentration is

$$
\bar{c}(\eta)=f(\eta) \frac{\varepsilon u(\eta)+1}{\varepsilon+1} .
$$

For $\theta=1$ we can evaluate (8.8) and obtain

$$
\bar{c}(\eta)=\frac{\varepsilon u(\eta)+1}{\varepsilon+1}\left(1-\frac{\log \left(\frac{\varepsilon u(\eta)+)}{\varepsilon+1}\right)}{\log \left(\frac{1}{\varepsilon+1}\right)}\right) .
$$




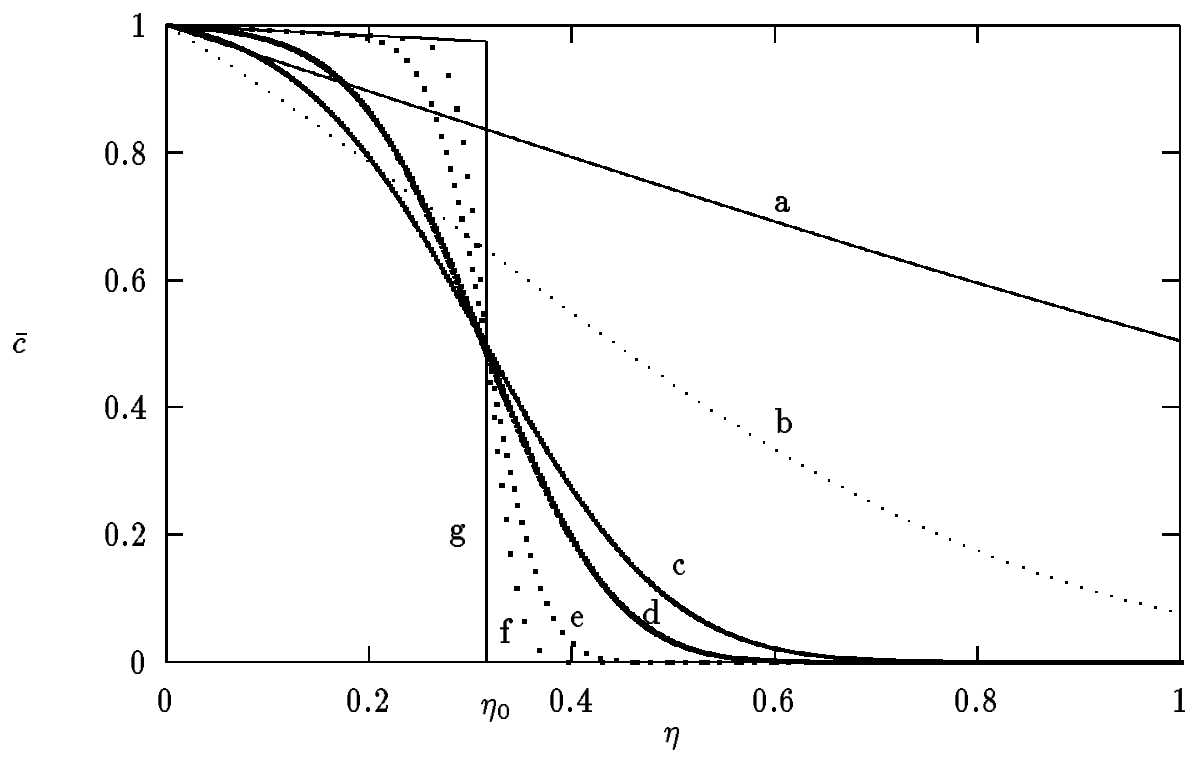

Figure 8. The scaled radionuclide concentration $\bar{c}(\eta)$ for $\varepsilon=0.2$ and different values of $\theta$ : a. $\theta=1.0$, b.

$$
\theta=0.1 \text {, c. } \theta=0.01 \text {, d. } \theta=0.005 \text {, e. } \theta=0.001 \text {, f. } \theta=0.0003 \text {, g. } \theta=0.0 \text {. }
$$

Figure 8 shows the scaled concentration $\bar{c}$ for different values of $\theta$ and $\varepsilon=0.2$. The results in this figure indicate that, as $\theta \rightarrow 0$, the concentration $\bar{c}$ has a discontinuity at $\eta=\eta_{0} \approx 0.316$ with $\bar{c}=0$ for $\eta>\eta_{0}$. This is a direct consequence of the limiting behavior of $(B V P)$ as $\theta \rightarrow 0$ : i.e.

$$
\left\{\begin{array}{l}
f^{\prime}\left(v-\frac{\eta}{2}\right)=0 \quad \text { for all } \eta>0 \\
f(0)=1, f(\infty)=0
\end{array}\right.
$$

which implies a piecewise constant solution

$$
f(\eta)=\left\{\begin{array}{llr}
1 & \text { for } & 0<\eta<\eta_{0} \\
0 & \text { for } & \eta>\eta_{0}
\end{array}\right.
$$

and thus

$$
\bar{c}(\eta)=\left\{\begin{array}{llr}
\frac{\varepsilon u(\eta)+1}{\varepsilon+1} & \text { for } & 0<\eta<\eta_{0} \\
0 & \text { for } & \eta>\eta_{0}
\end{array}\right.
$$

As a consequence a radionuclide front emerges which moves with speed $v\left(\eta_{0}\right) / \sqrt{t}$, where $v\left(\eta_{0}\right)=\max ((v(\eta))=$ $\eta_{\mathrm{o}} / 2$, see property (iii) in Section 5 . The position of the front in the $(z, t)$-plane is given by $s(t)=$ $2 v\left(\eta_{0}\right) \sqrt{t}=\eta_{0} \sqrt{t}$, which is equivalent to the path of a tracer particle released at $t=0$ in $z=0$, i.e. at the beginning of the brine transport process. Hence in the limit $\theta \rightarrow 0$, the movement of the tracer is caused by the compressibility effect only.

\section{Discussion}

Self-similar solutions make the analysis tractable and (semi-)explicit results can be obtained. A crucial requirement for the applicability of similarity arguments is that both the governing equations and initial and/or boundary conditions be reducible to similarity form. Hence, only in special cases one may expect to find similarity solutions. Due to the piecewise initial density data in Problem I and II we may consider the obtained similarity solutions as upper limits for the compressibility effect. Smooth initial data decrease the magnitude of the enhanced flow.

In analyzing Problems I and II we show explicitly the effect of enhanced groundwater flow due to compressibility of fluid caused by high salt concentration gradients. Characteristic for both problems 
is the occurrence of additional specific discharge in a direction perpendicular to the main groundwater direction. In both problems $q_{z}$ is not influenced by gravity. The only quantity that depends on gravity is the specific discharge in the $y$-direction, parallel to the main flow $q_{f}$.

In this paper we only consider constant (molecular) diffusion. In a more realistic description a velocity dependent dispersion matrix has to be introduced into equation (2.2). Molecular diffusion underestimates the compressibility effects.

When transversal dispersion, due to the (regional) back ground flow $q_{y}$, dominates the dispersion/diffusion in the $z$-direction we arrive at a different situation. Under the assumption $\left|q_{z}\right|<<\left|q_{y}\right|$ (2.5) reduces to

$$
\begin{array}{r}
D \approx \phi D_{\mathrm{mol}}+\alpha_{T}\left|q_{y}\right|= \\
\phi D_{\mathrm{mol}}+\alpha_{T}\left|q_{f}-\frac{\kappa}{\mu}\left(\rho-\rho_{f}\right) g \sin \beta\right|,
\end{array}
$$

yielding $D$ as a function of $\rho$. The analysis of this case is quite different from what we discussed here and will be published elsewhere [Van Duijn \& Schotting ,1996].

If $q_{f}>>\frac{\kappa}{\mu}\left(\rho_{s}-\rho_{f}\right) g \sin \beta$ we may replace (9.2) by

$$
D \approx \phi D_{\mathrm{mol}}+\alpha_{T}\left|q_{f}\right|,
$$

yielding a constant dispersivity which is in general much larger then the effective molecular diffusivity $\phi D_{\mathrm{mol}}$. The number

$$
P=\frac{\alpha_{T}\left|q_{f}\right|}{\phi D_{\mathrm{mol}}}
$$

indicates the relative importance of (transversal) dispersion with respect to molecular diffusion. If $P<1$ diffusion dominates dispersion. When $q_{f}=100 \mathrm{~m} / \mathrm{yr}$ typical values of the dispersivity are: $D\left(\alpha_{T}=0.1\right.$ $\mathrm{m}) \approx 3.10^{-7} \mathrm{~m}^{2} / \mathrm{s}$ and $D\left(\alpha_{T}=1.0 \mathrm{~m}\right) \approx 3.10^{-6} \mathrm{~m}^{2} / \mathrm{s}$. The corresponding $P$-numbers are: $\approx 10^{3}$ and $\approx 10^{4}$. It is obvious that such values of the dispersivity will enhance the compressibility effects in brine transport.

If we consider longitudinal dispersion of radionuclides in the $z$-direction (2.5) reduces to

$$
D\left(q_{z}\right)=D_{c}+\alpha_{L}\left|q_{z}\right|,
$$

remembering that $D_{c}=\phi D_{c-m o l}=\theta D$. By virtue of the $1 / \sqrt{t}$-decay of $q_{z}(z, t)$ one expects the longitudinal dispersion to dominate diffusion only on the short time scale in Problem II. A rough estimate of the order of magnitude of the time $\hat{t}$ after which diffusion becomes dominant can be given by using the approximation $q_{z}(z, t) \approx v_{\varepsilon}(0) / \sqrt{t}$ in

$$
P=\frac{\alpha_{L}\left|q_{z}(z, t)\right|}{D_{c}}<1
$$

hence

$$
\hat{t} \approx\left(\frac{\alpha_{L} \varepsilon}{\gamma}\right)^{2} \frac{\phi}{\pi \theta D} .
$$

Typical values of $\hat{t}$ are (for $\theta=1): \hat{t}\left(\alpha_{l}=1 \mathrm{~m}\right) \approx 0.9$ yr and $\hat{t}\left(\alpha_{L}=10 \mathrm{~m}\right) \approx 88 \mathrm{yr}$, where as the typical time scale of brine transport processes is usually in the order of thousands of years. Hence at a very early stage of the brine transport process molecular diffusion starts to dominate longitudinal dispersion. Notice that $\hat{t}$ decreases when we replace the effective molecular diffusivity by a constant transversal diffusivity $\alpha_{T} q_{f}$, as discussed above.

\section{Conclusions}

1. Compressibilty effects in brine transport in porous media are small and have in most practical cases only little effect on the density distributions. We studied two specific brine problems and compared the solutions for $\varepsilon>0$ with the corresponding Boussinesq solutions for $\varepsilon \rightarrow 0$, where $\varepsilon$ denotes the relative density difference. 
2. We found that high salt concentration gradients induce a convective flux, which is perpendicular to the main groundwater flow direction in the problems studied in this paper. The magnitude of this flux depends upon the relative density difference and the effective diffusivity/dispersivity of salt.

3. Taking only molecular diffusion into account underestimates the convective brine transport perpendicular to the main flow direction. For the problems studied in this paper it is more realistic to replace the molecular diffusivity by the transversal dispersivity due to the (regional) background flow. This increases the magnitude of $q_{z}$ significantly and $q_{z}$ can no longer be neglected as convective transport mechanism.

4. The similarity solutions presented are both of practical and theoretical use. First they provide us with detailed qualitative and quantitative information about the nature of compressibility effects. Secondly the solutions can be used to verify the accuracy of numerical codes designed to simulate brine transport.

5. The similarity solutions have the following properties: i) $u^{\prime}(\eta)<0$ for all $-\infty<\eta<+\infty$ (Problem I) and $0 \leq \eta<+\infty$ (Problem II), ii) there exists a number $\eta_{0}$ such that $u^{\prime \prime}(\eta)<0$ for $\eta<\eta_{0}$ and $u^{\prime \prime}(\eta)>0$ for $\eta>\eta_{0}$, iii) $v\left(\eta_{0}\right)=\eta_{0} / 2$ and $v^{\prime}(\eta)>0$ for $\eta<\eta_{0}$ and $v(\eta)<0$ for $\eta>\eta_{0}$. The number $\eta_{0}$ plays a prominent role in the simultaneous transport of radionuclides.

6. The results of the asymptotic analysis can be used to approximate the similarity solutions up to a given accurracy.

7. When considering simultaneous transport of brine and dissolved radionuclides we can construct an explicit solution for the radionuclide mass fraction expressed in terms of the solution of the underlying brine problem.

8. In the limit of vanishing radionuclide diffusion/dispersion a radionuclide front emerges in Problem II which travels with speed $v\left(\eta_{0}\right) / \sqrt{t}$. Hence, the movement of the radionuclide is caused by the compressibility effect only.

\section{Acknowledgments}

We acknowledge S.M. Hassanizadeh (Delft University of Technology, Faculty of Civil Engineering) and T. Leijnse (National Institute of Public Health and Environment Protection (RIVM) in Bilthoven), for bringing this subject to our attention.

\section{References}

[1] Bear,J., Dynamics of fluids in porous media, Elsevier, New York,(1972)

[2] Bornemann,O \& R. Fischbeck, Ablaugung und Hutgesteinsbildung am Salzstock Gorleben, Z. Geol. Ges., 137 (1986), 71-83

[3] Carey, A.E., S.W. Wheatcraft, R.J. Glass \& J.P. O'Rourke, Non-fickian ionic diffusion across high concentration gradients, Water Res. Research, vol 31, 9 (1995) 2213-2218.

[4] Duijn, C.J. van \& L.A. Peletier, A class of similarity solutions of nonlinear diffusion equations, Arch. Rational Mech. Anal. 65 (1977) 363-377.

[5] Duijn, C.J. van, L.A.Peletier \& R.J.Schotting, On the analysis of brine transport in porous media, European. Journ. of Appl.Math.1992.

[6] Duijn, C.J. van \& F.J.T Floris, Mathematical analysis of the influence of power law fluid rheology on a capillary diffusion zone, submitted to J. of Petr. Sc \& Eng. (1992)

[7] Duijn, C.J.van, S.M.Gomez \& Hongfei Zhang, On a class of similarity solutions of the equation $u_{t}=\left(|u|^{m-1} u_{x}\right)_{x}$ with $m>-1$, IMA Journal of Appl. Math.41 (1988) 147-163. 
[8] Van Duijn, C.J. \& R.J. Schotting, Brine transport in porous media: On the use of Von Mises and similarity solutions, submitted for publication (1996)

[9] Esteban, J.R., A. Rodriquez \& J.L. Vazquez, A nonlinear heat equation with singular diffusivity, Commun. in Part. Diff. Eq., 13 (8) (1988), 985-1039.

[10] Glasbergen, P., Extreme Salt Concentrations in Deep Aquifers in the Netherlands, The Science of the Total Environment,21 (1981), 251-259

[11] Hassanizadeh, S.M. \& T.Leijnse, On the modelling of brine transport in porous media, Water Resources Research 24(1988) 321-330

[12] Hassanizadeh, S.M., Experimental study of coupled flow and mass transport: a model validation exercise, ModelCARE 90: Calibration in Groundwater Modelling (Proceedings of the conference held in The Hague, September 1990, IAHS Publ. no. 195 (1990)

[13] Hassanizadeh, S.M. \& T. Leijnse, A non-linear theory of high-concentration-gradient dispersion in porous media, Submitted and accepted for publication in Adv. Water Res. Research (1995)

[14] Herbert, A.W., C.P.Jackson \& D.A.Lever, Coupled Groundwater Flow and Solute Transport With Fluid Density Strongly Dependent Upon Concentration, Water Res. Research 24(1988) 1781- 1795

[15] Josseling de Jong, G. de \& C.J.van Duijn, Transverse dispersion from an originally sharp fresh-salt interface caused by shear flow, Journal of Hydrology 84 (1986) 147-163

[16] Maple, Version MapleV Release 3, Computer algebra package (software), Waterloo Maple Software, Canada (1994)

[17] Oldenburg, C.M. \& K. Pruess, Dispersive transport dynamics in a strongly coupled groudwater-brine flow system, Water Res. Research 31(2) (1995), 289-302.

[18] Mises, R. von \& K.O. Friedrichs, Fluid dynamics, Applied Mathematics Series 5, Springer, New York (1973), 171.

[19] Taylor, G., Dispersion of soluble matter in solvent flowing slowly through a tube, Proc. Roy. Soc. London (1953) 219, 186-203.

[20] Taylor, G., Conditions under which dispersion of a solute in a stream can be used to measure molecular diffusion, Proc. Roy, Soc. London (1954) 225, 473-477.

[21] Roxburgh, I.S., Geology of high-level nuclear waste disposal - An introduction, Chapman and Hall Ltd, London (1987)

[22] Swedish Nuclear Inspectorate HYDROCOIN, An international project for studying groundwater hydrology modeling strategies, Level 1 Final Report: Verification of groundwater flow models, Case 5, Swedish Nucl.Insp.,Stockholm (1986)

[23] Weast, R.C., Handbook of Chemistry and Physics, 63rd ed., CRC Press Boca Raton Fla.1982, p. D261

[24] Visser, W.A., Waste Disposal and Underground Waters, Geologie en Mijnbouw 53 (1974) 6, 249-256 